\title{
Treating Subthreshold Depression in Primary Care: A Randomized Controlled Trial of Behavioral Activation With Mindfulness
}

Samuel Y. S. Wong, $M D^{1}$

Yu Ying Sun, $P b D^{1}$

Aaroy T. Y. Chan, $\mathrm{MPH}^{1}$

Maria K. W. Leung, MBA, MFM, FHKAM, FHKCFP, FRACGP, $M R C G P^{2}$

David V. K. Chao, MFM, FRCGP, FHKAM $^{3}$

Carole C. K. Li, $P_{s y D^{4}}$

King K. H. Chan, MFM, MRCGP, FRACGP, FHKAM ${ }^{5}$

Wai Kwong Tang, $M D^{6}$

Trevor Mazzucchelli, $\mathrm{PbD}^{7}$

Alma M. L. Au, PbD

Benjamin H. K. Yip, MS, PbD

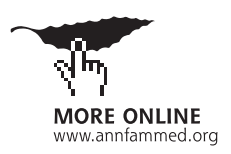

Conflicts of interest: authors report none.

\section{CORRESPONDING AUTHOR}

Samuel Y. S. Wong, MD

4/F, The Jockey Club School of Public Health and Primary Care Building, Prince of Wales Hospital

The Chinese University of Hong Kong 30-32 Ngan Shing St, Sha Tin New Territories, Hong Kong yeungshanwong@cuhk.edu.hk

\begin{abstract}
PURPOSE We undertook a randomized controlled trial to assess the efficacy of group-based behavioral activation with mindfulness (BAM) for treating subthreshold depression in primary care in Hong Kong.

METHODS We recruited adult patients aged 18 years or older with subthreshold depression from public primary care clinics and randomly assigned them to a BAM intervention group or a usual care group. The BAM group was provided with eight 2-hour weekly BAM sessions by trained allied health care workers. Patients in the usual care group received usual medical care with no additional psychological interventions. The primary outcome was depressive symptoms measured by the Beck Depression Inventory-II at 12 months. Secondary outcomes included incidence of major depressive disorder at 12 months. We assessed quality of life, activity and circumstances change, functional impairment, and anxiety at baseline, end of intervention, 5 months, and 12 months.
\end{abstract}

RESULTS We randomly allocated 115 patients to the BAM intervention and 116 patients to usual care. At 12 months, compared with usual care peers, BAM patients had a slightly more favorable change in levels of depressive symptoms on the Beck Depression Inventory-II (between-group mean difference in score $=-3.85 ; 95 \% \mathrm{Cl},-6.36$ to -1.34 ; Cohen $d=-0.46,95 \% \mathrm{Cl},-0.76$ to $-0.16)$. Incidence of major depressive disorder was lower with BAM $(10.8 \%$ vs $26.8 \%, P=.01)$, whereas groups did not differ significantly on other secondary outcomes at 12 months.

CONCLUSIONS Group BAM appears to be efficacious for decreasing depressive symptoms and reducing the incidence of major depression among patients with subthreshold depression in primary care, although generalizability of our findings may be limited.

Ann Fam Med 2018;16:111-119. https://doi.org/10.1370/afm.2206.

\section{INTRODUCTION}

$\mathrm{T}$ he lifetime prevalence of subthreshold depression is estimated to be $10 \%$ to $24 \%,{ }^{1,2}$ and this condition is common in primary care settings. ${ }^{3}$ Subthreshold depression can cause functional impairment ${ }^{4}$ and considerable economic costs, ${ }^{5}$ and it is a strong risk factor for developing major depressive disorder. ${ }^{6}$

According to the quality standard for depression care in adults from the National Institute for Health and Care Excellence, people with persistent subthreshold depressive symptoms or mild to moderate depression should receive appropriate low-intensity psychosocial interventions. ${ }^{7}$ Behavioral activation is a brief and simple structured treatment for depression that aims to activate individuals in specific ways that will increase rewarding experiences in their lives. ${ }^{8}$ A series of meta-analyses concluded that behavioral activation was effective for treating moderate to severe depression, ${ }^{9-12}$ and it may be preferable in primary care settings because it can be delivered by health professionals with less training. ${ }^{13}$ Recently, a 
large study showed that behavioral activation can be delivered by junior mental health workers. ${ }^{14}$ Although several studies have examined the effectiveness of behavioral activation, few have assessed its effects among people with subthreshold depression. Moreover, although the developers of behavioral activation have suggested and encouraged therapist use of mindfulness as a therapeutic method to reduce rumination in depressed patients, ${ }^{15}$ no large study has explicitly combined behavioral activation techniques with mindfulness skills and evaluated their combined effectiveness in reducing depressive symptoms. ${ }^{16}$ Only a small pilot study, having 16 patients, has combined behavioral activation and mindfulness to improve well-being of adults outside health care settings. ${ }^{17}$

In Hong Kong primary care settings, $40 \%$ to $60 \%$ of patients are older adults who have multiple chronic conditions ${ }^{18}$ and are at risk of having subthreshold depression and developing major depression. ${ }^{19}$ Currently, there is no study in a Chinese population evaluating the effectiveness of group behavioral activation in the treatment of subthreshold depression. The aim of this study was to evaluate the effectiveness of group behavioral activation with a distinct mindfulness component in treating subthreshold depression among adults in public primary care in Hong Kong.

\section{METHODS}

\section{Patients}

Patients were recruited from 16 general outpatient clinics in the New Territories East Cluster (8 clinics), Kowloon East Cluster (6 clinics), and Kowloon Central Cluster (2 clinics) between September 2014 and December 2015. The inclusion criteria were an age of 18 years or older, ability to speak Cantonese, and presence of subthreshold depression, defined as a score of 5 to 9 on the 9-item Patient Health Questionnaire (PHQ-9) depression scale. ${ }^{20-22}$ Exclusion criteria were dysthymia with subthreshold depressive symptoms that had lasted for 2 years or more; major depression within the past 6 months; lifetime history of other psychiatric disorders (generalized anxiety disorder, psychosis, schizophrenia, or bipolar affective disorder) ${ }_{i}$ alcohol or substance abuse ${ }_{i}$ presence of serious suicidal risk; medical illness with a prognosis of less than 6 months to live; current receipt of treatments (antidepressants or other psychotropic medications, or enrollment in any form of psychological interventions) for any depressive disorders or symptoms; and inability to read or write.

Patients provided written consent. This study was approved by the Joint Chinese University of Hong Kong-New Territories East Cluster Clinical Research
Ethics Committee (CREC Ref No.: CRE-2012.583). The clinical registry number was ChiCTR-TCS-14004601.

\section{Procedure}

Randomization was conducted after patients returned a baseline questionnaire. A statistician who was not involved in any part of the study independently randomized patients by using a predetermined random table generated by Microsoft Excel 2003 (Microsoft Corporation). Patients were randomly assigned to 2 treatment arms: behavioral activation with mindfulness (BAM) and usual care. The sealed randomization number was not decoded until the patient was assigned to its corresponding randomization number. The allocation was concealed from the researchers, research assistants, and coordinators who carried out the baseline assessment or recorded the data.

\section{Group BAM Intervention}

We used the BAM interventions for depression and well-being ${ }^{17}$ because of their established effectiveness. A treatment outline was retrieved from the workbook and adapted for the Chinese culture (Supplemental Appendix, available at http://www.annfammed.org/ content/16/2/111/suppl/DC1/). The final intervention protocol was established after conduct of a pilot study. The intervention consisted of weekly 2 -hour sessions for 8 consecutive weeks. The first 4 sessions entailed psychoeducation with respect to well-being; setting short- and long-term goals; self-monitoring of activity and mood using activity logs to recognize the association between specific activities and mood; scheduling daily activities; and identifying avoidance and its impact to allow patients to be more aware of their decision making. Sessions 5 through 7 included a 0.5 -hour behavioral activation review and a 1.5 -hour mindfulness practice that consisted of training on basic mindfulness skills including body scan and both sitting and walking meditation. Mindfulness practice was consolidated to make the patients cultivate attention on their behavioral patterns. All patients were provided with a compact disc including 6 audio recordings of guided meditations for home practice (body scan, sitting meditation, lake meditation, and mountain meditation). The home practice was expected to be 10 minutes/day on at least 6 days/week after session 5 , increasing to 20 minutes/day after session 6 , and increasing further to 45 minutes/day after session 7 so that there was an incremental increase of practice time, which may facilitate adaptation to these activities. The last session, session 8 , aimed to review the previous skills learned and to reinforce these skills in daily life.

The BAM patients were divided into 9 training cohorts for the intervention. Four allied health profes- 
sionals (1 nurse, 3 social workers) served as instructors. None had prior training in behavioral activation or mindfulness. All received 32 hours of training and 8 hours of supervision by 2 experienced clinical psychologists with more than 4 years of experience in behavioral activation plus 1 clinical psychologist who was an experienced mindfulness instructor and had taught mindfulness-based programs for more than 10 years.

\section{Usual Care}

Generally, patients with subthreshold depression in primary care were not referred for mental health intervention but received active monitoring or regular minimal counseling from primary care physicians. Patients assigned to the usual care group continued to receive their usual medical care from physicians in the general outpatient clinics and were allowed unrestricted access to medical care for their concerns about depression or anxiety. Our research team did not provide any additional mental health intervention to patients in this group.

\section{BAM Intervention Fidelity Assessment}

Two clinical psychologists independently assessed 2 aspects of fidelity of the BAM intervention: adherence and competence. We assessed $12.5 \%$ of the treatment courses. The number of adherence items ranged from 7 to 11, depending on the outline of the sessions. Response options ranged from 0 (not covered at all) to 2 (adequately covered) or 3 (covered thoroughly). As there was no consensus about the definition of good adherence to BAM, we defined high treatment fidelity as the rating of at least $80 \%$ of items as adequately covered or thoroughly covered. We developed the competence measure from a fidelity guide of group cognitive behavioral therapy ${ }^{23}$ and covered the assessment of the BAM intervention and group engagement. Fourteen items were rated on a 7 -point scale (0 to 6), with an average score of 4.0 indicating competent BAM delivery.

\section{Outcomes}

The trial's primary outcome was reduction of depressive symptoms at 12 months. It was measured by a validated Chinese version of the Beck Depression Inventory-II (BDI-II) that included 21 items, with possible total scores ranging from 0 to $63 .{ }^{24-26}$

A secondary outcome was the incidence of major depressive disorder defined by Diagnostic and Statistical Manual of Mental Disorders, 4th Edition (DSM-IV) criteria. Presence of major depressive disorder was ascertained by research assistants with a master's degree in counseling using the Structured Clinical Interview for DSM-IV Axis I Disorders (SCID). ${ }^{27}$ The research assistants were blinded to the patient's group allocation.
We assessed 5 other secondary outcomes. (1) The validated Chinese version of the 12 -item Medical Outcomes Study Short-Form Health Survey (SF-12) assessed health-related quality of life, including both physical and mental functioning, and well-being. ${ }^{28}$ (2) The 8-item Activity and Circumstances Change Questionnaire (ACCQ) ${ }^{16}$ assessed the extent to which patients experienced both activity change and circumstantial change. For each item, patients responded on a 5 -point Likert scale with 1 indicating no positive change and 5 indicating great positive change. (3) The Sheehan Disability Scale (SDS) ${ }^{29}$ was used to allow patients to rate their impairment in 3 life domains (work, social, and family/home management) using a scale from 0 to 10 , where 0 indicates no impairment and 10 indicates extreme impairment. (4) The State Trait Anxiety Inventory (STAI) ${ }^{30}$ was used to measure anxiety symptoms. Based on a 4 -point Likert scale, the inventory has 2 separate components: state anxiety (STAI-S) and trait anxiety (STAI-T). (5) A self-reported treatment evaluation was conducted immediately after the intervention to assess patient satisfaction. Six aspects (content, format, atmosphere, relationship with other group members, location and environment, and overall satisfaction) were captured on a scale from 1 , indicating "dissatisfied," to 5, indicating "very satisfied."

With the exception of major depressive disorder (assessed only at baseline and 12-month follow-up), all outcomes were assessed at baseline, end of intervention, the 5-month follow-up (ie, 3 months after intervention), and the 12-month follow-up (ie, 10 months after intervention).

\section{Statistical Methods}

We performed descriptive analyses of patients' baseline characteristics, including age, sex, marital status, educational background, medical history, and PHQ-9 score. For primary analyses, treatment outcomes were assessed by analysis of covariance (ANCOVA), with the baseline BDI-II scale score and other significantly different baseline characteristics as the covariates, and group as the factor. Analysis was performed on an intention-to-treat basis. We used a modified intention-to-treat sample by including all patients in the original group assignment of the random allocation with complete data (observed data). ${ }^{14}$ The effect size was calculated by Cohen $d .^{31,32}$ Effect sizes of $0.2,0.5$, and 0.8 were respectively defined as small, medium, and large. ${ }^{33}$

We also completed a sensitivity analysis to assess the effect of missing data using multiple imputation models. Multiple imputation was conducted by creating 20 complete data sets by chained equations under the assumption that data were missing at random $(\mathrm{R}$ package: MICE; version 2.2.5; R Project for Statistical 
Computing). ${ }^{34}$ We adopted the Rubin rule to calculate the effect estimates from the imputed data sets. ${ }^{35} \mathrm{We}$ also did a per-protocol analysis by including those patients who completed their treatment as originally assigned: those in the BAM group who attended at least 6 sessions and those in the usual care group who returned questionnaires and stayed in the group. ${ }^{36}$

We compared the incidence of major depressive disorder between groups. $P$ values were computed by logistic regression analysis with group and other significantly different baseline characteristics as covariates. In our sensitivity analysis, we also assessed the effect of BAM training cohort and location (as the fixed covariate). An effect of the BAM instructor was tested by ANOVA (only with data from the intervention group), with primary time point (12 months) as the outcome and instructor as the group effect.

\section{RESULTS}

About $11 \%$ of the patients in primary care clinics $(1,619$ of 15,199$)$ were identified as eligible for the study, and $14 \%$ of these eligible patients $(231$ of 1,619$)$ agreed to participate (Figure 1). A total of 115 patients were assigned to the BAM group and 116 patients were assigned to the usual care group. In the modified intention-to-treat population, $90.4 \%$ of the patients in the BAM group (104 of 115) and $98.3 \%$ in the usual care group (114 of 116), were assessed at the end of the intervention period, and $71.3 \%$ (82 of 115 ) and $87.1 \%$ (101 of 116$)$, respectively, were assessed at 12 months.

The large majority of patients were female $(93.1 \%)$, housewives or retired $(61.9 \%)$, and married (72.8\%) (Table 1). The mean age was 54.0 years. Except for marital status and cancer history, demographic characteristics were balanced between the 2 groups.

\section{Attrition and Attendance}

Overall, $85.2 \%$ of patients in the BAM group (98 of 115) attended at least 1 intervention session. Six patients did not attend any sessions, although they returned questionnaires at the end of the intervention. On average, patients attended 6.1 sessions out of the 8 sessions offered. In the per-protocol analysis, 66 patients $(67.3 \%)$ in the BAM group and 98 patients $(84.5 \%)$ in usual care group were evaluated. There were no significant differences in the patient characteristics among those patients who were included in the perprotocol analysis and those patients who were not.

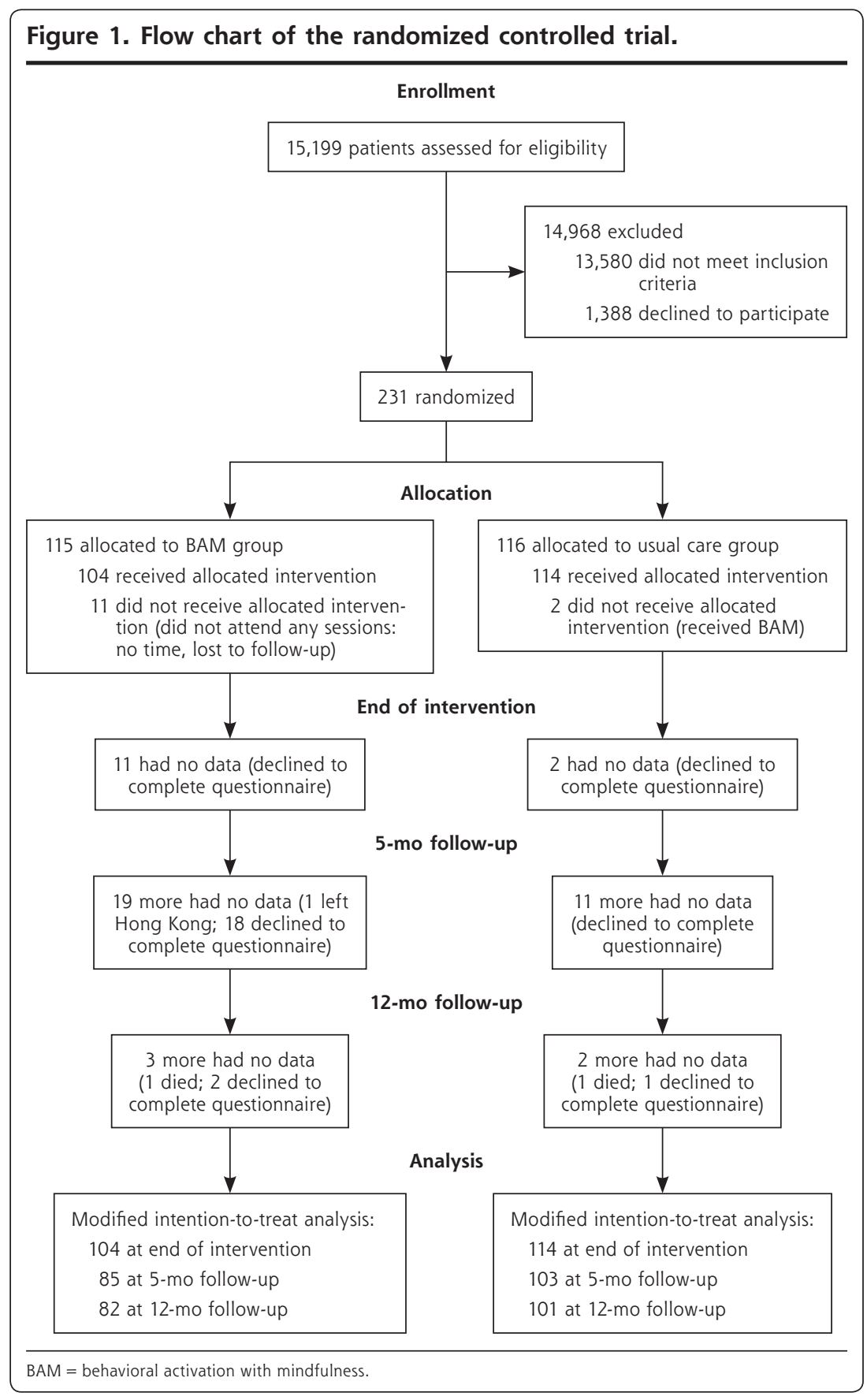


Depressive Symptoms and Secondary Outcomes

Table 2 shows the results of our analysis of the primary outcome and secondary outcomes. At the end of the intervention, compared with the usual care group, the BAM group had a significantly more favorable change in level of depressive symptoms as assessed with the BDI-II scale (between-group mean difference $=-3.57 ; 95 \%$ $\mathrm{CI},-5.38$ to -1.78 ; Cohen $d=-0.54 ; 95 \% \mathrm{CI},-0.82$ to -0.27 ). The BAM group also had greater improvements in quality of life for the mental component summary score (between-group mean difference $=3.22 ; 95 \% \mathrm{CI}$, 1.09 to 5.35 ; Cohen $d=0.41 ; 95 \% \mathrm{CI}, 0.14$ to 0.69 ) and ACCQ score (between-group mean difference $=2.62$; $95 \% \mathrm{CI}, 1.13$ to 4.11 ; Cohen $d=0.48 ; 95 \% \mathrm{CI}, 0.21$ to $0.75)$. There was a trend toward a reduction in anxiety assessed with the STAI-S scale favoring the BAM group as well (between-group mean difference $=-2.18 ; 95 \%$ $\mathrm{CI},-4.44$ to -0.08 ; Cohen $d=-0.26 ; 95 \% \mathrm{CI},-0.54$ to -0.01 ). The estimated marginal means for the primary

Table 1. Characteristics of Patients Overall and by Group

\begin{tabular}{|c|c|c|c|c|}
\hline Characteristic & $\begin{array}{c}\text { Total } \\
(\mathrm{N}=231)\end{array}$ & $\begin{array}{l}\text { BAM Group } \\
(n=115)\end{array}$ & $\begin{array}{l}\text { Usual Care } \\
\text { Group } \\
(n=116)\end{array}$ & $\begin{array}{c}\text { Test } \\
\text { Statistic, } \\
t \text { or } \chi^{2}\end{array}$ \\
\hline Age, mean (SD), y & $54.0(12.0)$ & $55.3(10.4)$ & $52.7(13.3)$ & 1.676 \\
\hline \multicolumn{5}{|l|}{ Sex, No. (\%) } \\
\hline Male & $16(6.9)$ & $6(5.2)$ & $10(8.6)$ & \multirow[t]{2}{*}{1.038} \\
\hline Female & $215(93.1)$ & $109(94.8)$ & $106(91.4)$ & \\
\hline \multicolumn{5}{|l|}{ Education, No. (\%) } \\
\hline Primary or less & $72(31.2)$ & $36(31.3)$ & $36(31.0)$ & \multirow[t]{3}{*}{0.423} \\
\hline Middle school & $113(48.9)$ & $58(50.4)$ & $55(47.4)$ & \\
\hline Diploma or more & $46(19.9)$ & $21(18.3)$ & $25(21.6)$ & \\
\hline \multicolumn{5}{|l|}{ Occupation, No. (\%) } \\
\hline Housewife & $89(38.5)$ & $44(38.3)$ & $45(38.8)$ & \multirow[t]{4}{*}{1.176} \\
\hline Retired & $54(23.4)$ & $24(20.9)$ & $30(25.9)$ & \\
\hline Employed or self-employed & $74(32.0)$ & 39 (33.9) & $35(30.2)$ & \\
\hline Part-time or other & $14(6.1)$ & $8(7.0)$ & $6(5.2)$ & \\
\hline \multicolumn{5}{|l|}{ Marital status, No. (\%) } \\
\hline Single & $38(16.7)$ & $16(14.0)$ & $22(19.3)$ & \multirow[t]{3}{*}{$8.899^{a}$} \\
\hline Married & $166(72.8)$ & $92(80.7)$ & $74(64.9)$ & \\
\hline Live alone & $24(10.5)$ & $6(5.3)$ & $18(15.8)$ & \\
\hline \multicolumn{5}{|l|}{ Income, No. (\%) } \\
\hline$<\$ 5,000$ & $75(33.2)$ & $39(34.5)$ & $36(31.9)$ & \multirow[t]{4}{*}{1.128} \\
\hline$\$ 5,000-\$ 10,000$ & $51(22.6)$ & $24(21.2)$ & $27(23.9)$ & \\
\hline$\$ 10,000-\$ 20,000$ & $74(32.7)$ & $35(31.0)$ & $39(34.5)$ & \\
\hline$>\$ 20,000$ & $26(11.5)$ & $15(13.3)$ & $11(9.7)$ & \\
\hline PHQ-9 score, mean (SD) & $7.35(1.41)$ & $7.38(1.40)$ & $7.32(1.43)$ & 0.342 \\
\hline \multicolumn{5}{|l|}{ Health conditions, No. (\%) } \\
\hline Diabetes & $26(11.3)$ & $15(13.0)$ & $11(9.5)$ & 0.733 \\
\hline Hypertension & $91(39.4)$ & $51(44.3)$ & $40(34.5)$ & 2.354 \\
\hline Rheumatoid arthritis & $30(13.0)$ & $16(13.9)$ & $14(12.1)$ & 0.174 \\
\hline Cancer & $18(7.8)$ & $13(11.3)$ & $5(4.3)$ & $3.932^{\mathrm{a}}$ \\
\hline
\end{tabular}

and secondary outcomes at the end of the intervention and follow-up are given in Supplementary Figures 1 through 7 (available at http://www.annfammed.org/ content/16/2/111/suppl/DC1/).

At the 5-month follow-up, there was still a significantly lower level of depressive symptoms in the BAM group as compared with the control group $(P=.015)$. At the 12-month follow-up, BAM had a persistent albeit small benefit over usual care in reducing depressive symptoms (between-group mean difference $=-3.85 ; 95 \% \mathrm{CI},-6.36$ to -1.34 ; Cohen $d=-0.46$; $95 \% \mathrm{CI},-0.76$ to -0.16$)$ and was associated with a lower incidence of major depressive disorder $(10.8 \%$ vs $26.8 \% ; P=.01$ ). There was no significant difference between groups for the other secondary outcomes. The treatment effects after adjusting for BAM training cohort or location were comparable to those seen in the primary analysis (Supplemental Table 1, available at http://www.annfammed.org/content/16/2/111/ suppl/DC1/), and there was no indication of an instructor effect $(\mathrm{F}=1.010, P=.39)$.

The analysis using imputed data showed a significant improvement in depressive symptoms at the end of the intervention $(P<.001)$ and at the 12 -month follow-up $(P=.01)$ in the BAM group, but there was only a trend in symptom change at the 5-month follow-up $(P=.051)$. In the per-protocol analysis, the BAM group had significantly lower levels of depressive symptoms compared with the usual care group at the end of the intervention $(P=.001), 5$ months $(P=.012)$, and 12 months $(P=.003)$, findings consistent with those of the primary analysis.

\section{BAM Intervention Fidelity and Evaluation}

The BAM intervention was delivered according to the protocol. Fully $90 \%$ of items (43 out of 48 ) followed the protocol. Percentage of content delivered according to protocol was higher for the mindfulness-only sessions than for the behavioral activation sessions (91.7\% and $88.9 \%$, respectively). Clinical psychologists rated the 4 therapists as 
competent in delivering the treatment for both the behavioral activation sessions (mean $=4.47, \mathrm{SD}=0.46)$ and the mindfulness sessions ( mean $=4.12, \mathrm{SD}=0.43$ ).

Our evaluation of 5 aspects of the BAM course showed that the mean overall satisfaction level was 4.16 $(\mathrm{SD}=0.85)$. The satisfaction with course content and philosophy, class format, class atmosphere, relationship between class members, and class location and environment were, respectively, $3.98(\mathrm{SD}=0.92), 3.97$ $(\mathrm{SD}=0.86), 4.19(\mathrm{SD}=0.82), 4.09(\mathrm{SD}=0.91)$, and 4.21
$(\mathrm{SD}=0.83)$. More than $80 \%$ of the patients (79 of 98$)$ thought the instructor affected them positively. The majority $(60.0 \%)$ reported that they kept in touch with other group members after the intervention ended. Approximately $80 \%$ (77 of 98 ) thought the practice and homework helped reduce their depressive symptoms. The majority of patients indicated that they would keep practicing the learned skills in the future $(74.5 \% ; 73$ of 98$)$ and would recommend this intervention to others $(85.9 \% ; 85$ of 98$)$.

\section{Table 2. Outcomes in the BAM Group and Usual Care Group at Various Time Points}

\begin{tabular}{|c|c|c|c|c|c|c|c|}
\hline \multirow[b]{2}{*}{ Outcome } & \multirow[b]{2}{*}{$\begin{array}{l}\text { Time } \\
\text { Point }\end{array}$} & \multirow{2}{*}{$\begin{array}{c}\text { BAM Group, } \\
\text { Mean (SD) } \\
(n=115)\end{array}$} & \multirow{2}{*}{$\begin{array}{c}\text { Usual Care } \\
\text { Group, } \\
\text { Mean (SD) } \\
\text { (n=116) }\end{array}$} & \multicolumn{2}{|c|}{$\begin{array}{l}\text { Modified } \\
\text { Intention-to-Treat Analysis }\end{array}$} & \multicolumn{2}{|c|}{ Imputed Data Analysis } \\
\hline & & & & $\begin{array}{c}\text { Between-Group } \\
\text { Difference }(95 \% \mathrm{Cl})\end{array}$ & $\begin{array}{c}P \\
\text { Value }\end{array}$ & $\begin{array}{c}\text { Between-Group } \\
\text { Difference }(95 \% \mathrm{Cl})\end{array}$ & $\begin{array}{c}P \\
\text { Value }\end{array}$ \\
\hline \multirow[t]{4}{*}{ BDI-II score ${ }^{a, b}$} & TO & $19.18(8.35)$ & $17.68(9.52)$ & - & & - & \\
\hline & T1 & $15.41(9.53)$ & $17.28(9.83)$ & $-3.57(-5.38$ to -1.78$)$ & $<.001$ & $-3.21(-5.02$ to -1.40$)$ & $<.001$ \\
\hline & $\mathrm{T} 2$ & $15.04(10.29)$ & $16.57(10.47)$ & $-2.97(-5.35$ to -0.59$)$ & .02 & $-2.32(-4.65$ to 0.01$)$ & .051 \\
\hline & T3 & $14.45(10.40)$ & $16.90(10.84)$ & $-3.85(-6.36$ to -1.34$)$ & .003 & $-3.06(-5.48$ to -0.64$)$ & .01 \\
\hline \multirow[t]{4}{*}{ SF-12: PCS score sc $^{b, c}$} & TO & $40.19(8.66)$ & $41.75(9.75)$ & - & & - & \\
\hline & $\mathrm{T} 1$ & $40.16(8.86)$ & $41.26(9.96)$ & $0.22(-1.70$ to 2.13$)$ & .82 & $-0.03(-1.92$ to 1.86$)$ & .97 \\
\hline & $\mathrm{T} 2$ & $39.61(8.98)$ & $40.00(9.46)$ & $0.72(-1.68$ to 3.13$)$ & .55 & $0.21(-2.04$ to 2.45$)$ & .86 \\
\hline & T3 & $39.19(8.67)$ & $40.15(10.20)$ & $0.18(-2.30$ to 2.65$)$ & .89 & $-0.10(-2.44$ to 2.25$)$ & .94 \\
\hline \multirow[t]{4}{*}{ SF12: MCS score ${ }^{b, c}$} & TO & $40.24(8.81)$ & 40.99 (10.11) & - & & - & \\
\hline & $\mathrm{T} 1$ & $42.96(9.62)$ & $40.95(9.84)$ & $3.22(1.09$ to 5.35$)$ & .003 & $2.99(0.85$ to 5.13$)$ & .006 \\
\hline & $\mathrm{T} 2$ & $42.02(9.27)$ & $41.33(8.87)$ & $1.43(-1.05$ to 3.91$)$ & .26 & $1.12(-1.24$ to 3.48$)$ & .35 \\
\hline & T3 & $42.81(10.00)$ & $42.24(9.14)$ & $1.41(-1.22$ to 4.04$)$ & .29 & $1.12(-1.33$ to 3.58$)$ & .37 \\
\hline \multirow[t]{4}{*}{ ACCQ score ${ }^{d}$} & TO & $13.87(6.58)$ & $13.31(5.90)$ & - & & - & \\
\hline & $\mathrm{T} 1$ & $17.30(6.14)$ & $14.63(6.44)$ & $2.62(1.13$ to 4.11$)$ & .001 & 2.47 (0.98 to 3.97$)$ & .001 \\
\hline & $\mathrm{T} 2$ & $16.20(6.62)$ & $16.09(7.31)$ & $0.13(-1.75$ to 2.00$)$ & .90 & $0.42(-1.43$ to 2.28$)$ & .65 \\
\hline & T3 & 17.07 (7.11) & $16.11(7.26)$ & $1.01(-1.08$ to 3.09$)$ & .34 & $1.15(-0.87$ to 3.17$)$ & .26 \\
\hline \multirow[t]{4}{*}{ SDS score ${ }^{b, e}$} & TO & $11.70(9.23)$ & $11.03(8.68)$ & - & & - & \\
\hline & T1 & $11.58(9.39)$ & $10.87(8.69)$ & $0.24(-1.98$ to 2.46$)$ & .83 & $0.37(-1.80$ to 2.54$)$ & .74 \\
\hline & $\mathrm{T} 2$ & $9.14(8.14)$ & $9.92(8.59)$ & $-1.27(-3.63$ to 1.08$)$ & .29 & $-0.38(-2.59$ to 1.84$)$ & .74 \\
\hline & T3 & $10.58(9.30)$ & $11.15(9.91)$ & $-1.23(-3.83$ to 1.37$)$ & .35 & $-0.62(-2.99$ to 1.76$)$ & .61 \\
\hline \multirow[t]{4}{*}{ STAI-S score ${ }^{f}$} & TO & $48.03(9.42)$ & $47.71(10.17)$ & - & & - & \\
\hline & $\mathrm{T} 1$ & $46.26(11.54)$ & $47.68(9.33)$ & $-2.18(-4.44$ to -0.08$)$ & .059 & $-1.87(-4.15$ to 0.41$)$ & .11 \\
\hline & $\mathrm{T} 2$ & $46.27(10.54)$ & $47.37(9.41)$ & $-1.64(-4.17$ to 0.89$)$ & .20 & $-1.30(-3.73$ to 1.13$)$ & .29 \\
\hline & T3 & $45.99(10.43)$ & $47.70(10.56)$ & $-2.50(-5.20$ to 0.19$)$ & .07 & $-1.72(-4.30$ to 0.86$)$ & .19 \\
\hline \multirow[t]{4}{*}{ STAI-T score } & TO & $47.76(8.18)$ & $46.87(8.70)$ & - & & - & \\
\hline & $\mathrm{T} 1$ & $46.75(9.38)$ & $47.43(8.15)$ & $-1.56(-3.48$ to 0.36$)$ & .11 & $-1.25(-3.13$ to 0.63$)$ & .19 \\
\hline & $\mathrm{T} 2$ & $46.47(8.92)$ & $47.22(8.52)$ & $-1.69(-3.87$ to 0.49$)$ & .13 & $-1.15(-3.18$ to 0.88$)$ & .26 \\
\hline & T3 & $47.17(8.97)$ & $48.14(8.48)$ & $-1.99(-4.27$ to 0.30$)$ & .09 & $-1.40(-3.65$ to 0.84$)$ & .22 \\
\hline
\end{tabular}

$\mathrm{ACCQ}=$ Activity and Circumstantial Change Questionnaire; BAM = behavioral activation with mindfulness; BDI-II: Beck Depression Inventory-II; MCS = mental component summary; PCS = physical component summary; SDS = Sheehan Disability Scale; SF-12 = 12-item Medical Outcomes Study Short-Form

Health Survey; STAI-S = State Trait Anxiety Inventory-State; STAI-T = State Trait Anxiety Inventory-Trait; T0 = baseline; T1 = end of intervention; T2 = 5-month follow-up; T3 = 12-month follow-up.

Note: Major depressive disorder at T3 was present in 9 patients $(10.8 \%)$ in the BAM group and 26 patients $(26.8 \%)$ in the usual care group $(P=.01)$. Analysis conducted using observed data, and $P$ value obtained by logistic regression analysis with cancer history and marital status as covariates.

a Possible range for total scores is 0 to 63 ; higher scores indicate more severe depression.

${ }^{b} P$ values for group effect were calibrated by analysis of covariance (ANCOVA) approach, with baseline value, cancer history, and marital status as covariates.

Possible range is 0 to 100; higher scores indicate better self-reported health.

d Possible range is 8 to 40 ; higher scores indicate greater positive change.

e Possible range is 0 to 40 ; higher scores indicate more functional impairment.

${ }^{f}$ Possible range is 20 to 80 ; higher scores indicate greater anxiety. 


\section{DISCUSSION}

We found that the BAM intervention had both shortterm and long-term effects in reducing depressive symptoms among patients with subthreshold depression in primary care. The between-group mean difference in score was 3.85 points on the BDI-II scale at the time of intervention completion, which was about $22 \%$ of the follow-up score in the usual care control arm and exceeds the $17.5 \%$ mean clinically important difference identified by Button et al. ${ }^{37}$ BAM still had a small benefit in reducing subthreshold depression at 12 months. Similar effect sizes for improvements have been reported for individual cognitive behavioral therapy, problem-solving therapy, interpersonal psychotherapy, and other individual psychological interventions. ${ }^{38}$ Generalizability of the BAM intervention to a broader population, such as patients with more severe symptoms or those with dysthymia, is possible but needs further research as our study included only patients who fulfilled the eligibility criteria and had greater representation of patients with certain characteristics (eg, female sex and occupation of housewife).

The incidence of major depressive disorder among people with subthreshold depression ranges from $8 \%$ to $47 \% .^{39-43}$ Strategies to prevent the onset of a major depressive episode can be highly effective. The combination of behavioral activation and mindfulness performed well in the prevention of major depressive disorder in our study: incidence in the BAM group was less than half that in the usual care group. BAM can be viewed as a preven- tive intervention for major depressive disorder among patients with subthreshold depressive symptoms who have not had a major depression episode within the past 6 months. Given the recurrent nature of major depressive disorder, it would be informative to further evaluate the response to BAM among those with chronic depression or dysthymia, as we excluded such patients from our study.

The objective of behavioral activation is to increase positive reinforcement and rewarding experiences in life. Although behavioral activation is action oriented while mindfulness emphasizes the acceptance and awareness of present moment emotions, thoughts, and bodily sensations, the two can be complementary. Previous research suggests that mindfulness practice could also generate positive emotions by cultivating self-compassion and self-confidence through an upward spiral process. ${ }^{44}$ We postulate that the maintenance of positive effects at 12 months could be related to the ongoing practice of mindfulness skills, as patients were given audio clips to promote ongoing practice.

The BAM intervention demonstrated short-term efficacy in improving quality of life, but only in the mental domain. It is possible that patients' physical condition and functional impairments are more difficult to change. Although some studies using either behavioral activation or mindfulness-based intervention have found significant improvement in quality of life, ${ }^{45,46}$ others have not. ${ }^{47,48}$

We found a marginally significant reduction of anxiety symptoms with BAM at the end of the intervention in our primary analysis. As the objective of this study was to evaluate the efficacy of BAM among patients with depressive symptoms, we do not know whether the same improvement could be observed among people with a principal diagnosis of anxiety disorders.

Fidelity assessments showed that the BAM intervention was delivered according to and adhered to the protocol, and allied mental health workers were competent in delivering behavioral activation as well as basic mindfulness training. These health workers received a total of 40 hours of training and clinical supervision. We suggest that allied health care workers have the capacity to deliver the BAM intervention effectively after structured training.

Our study has several limitations. First, it was impossible to conceal the group assignment after the first BAM session; thus, patients in the intervention group might have reported improvement because of a Hawthorne effect. Second, it was difficult to differentiate the efficacy of the behavioral activation and mindfulness components as we combined them. We did not distinguish the 2 components because we hoped to make the intervention a cohesive whole for future application in clinical practice, as it appears that the 2 
components may complement each other. But further recognition of the useful components is also important to understand the mechanism that is responsible for the effects observed. Third, generalizability of the intervention to a broader population might be limited given that a large share of patients declined to enroll in the study because of time conflicts and were unable to attend the 8 weekly sessions. This may be an issue especially among people with the hectic lifestyle often seen in developed cities, such as Hong Kong, potentially making it difficult for the working population to spare 2 hours a week for 8 consecutive weeks. The mildness of patients' depressive symptoms might have also played a role, as perhaps they felt that their symptoms were not severe enough for intervention. Fourth, the relatively low uptake rate and lower follow-up rate in the BAM group vs the usual care group may also raise issues regarding the implementation and feasibility of using BAM in real-life clinical practice. As the reasons for dropout were mainly related to schedule conflicts, however, and we did not find any significant difference in patient characteristics between those who did and did not drop out, modifications of the mode of BAM delivery may be needed to make it more acceptable to potential participants.

In conclusion, we have shown that BAM is a potentially feasible and efficacious intervention for reducing depressive symptoms and preventing major depression among people with subthreshold depression in primary care. As this intervention can be delivered by allied health care professionals with only about 1 week of training and can be implemented in primary care settings, we are conducting a cost-effectiveness and qualitative study to better understand the health service use implications, as well the potential mechanisms and relevant components acceptable to patients, to further improve this intervention and increase its reach among primary care patients.

To read or post commentaries in response to this article, see it online at http://www.AnnFamMed.org/content/16/2/111.

Key words: behavioral activation; mindfulness; subthreshold depression; prevention; primary care; randomized controlled trial

Submitted April 27, 2017; submitted, revised, August 22, 2017; accepted October 10, 2017.

Funding support: The authors received funding support from the Health and Medical Research Fund (HMRF), 11120501.

Acknowledgments: The authors would like to acknowledge the support of physicians, nurses, and assistant staff in the General Outpatient Clinics/ Family Medicine Center of the New Territories East Cluster, Kowloon East Cluster, and Kowloon Central Cluster in Hong Kong.

Author affiliations: The Jockey Club School of Public Health and Primary Care, The Chinese University of Hong Kong, Hong Kong, China
(Wong, Sun, T.Y. Chan, Yip); Fanling Family Medicine Centre, Hospital Authority, Hong Kong, China (Leung); United Christian Hospital, Hospital Authority, Hong Kong, China (Chao); Kowloon East Cluster, Hospital Authority, Hong Kong, China (Li); Kowloon Central Cluster, Hospital Authority, Hong Kong, China (K.H. Chan); Department of Psychiatry, The Chinese University of Hong Kong, Hong Kong, China (Tang); School of Psychology and Speech Pathology, Curtin University, Perth, Western Australia (Mazzucchelli); Department of Applied Social Sciences, The Hong Kong Polytechnic University, Hong Kong, China (Au)

Supplementary materials: Available at http://www.AnnFamMed. org/content/16/2/111/suppl/DC1/.

\section{References}

1. Kessler RC, Zhao S, Blazer DG, Swartz M. Prevalence, correlates, and course of minor depression and major depression in the National Comorbidity Survey. J Affect Disord. 1997;45(1-2):19-30.

2. Jongenelis K, Pot AM, Eisses AM, Beekman AT, Kluiter H, Ribbe MW. Prevalence and risk indicators of depression in elderly nursing home patients: the AGED study. J Affect Disord. 2004;83(2-3):135-142.

3. Rucci P, Gherardi S, Tansella M, et al. Subthreshold psychiatric disorders in primary care: prevalence and associated characteristics. J Affect Disord. 2003;76(1-3):171-181.

4. Backenstrass M, Frank A, Joest K, Hingmann S, Mundt C, Kronmüller KT. A comparative study of nonspecific depressive symptoms and minor depression regarding functional impairment and associated characteristics in primary care. Compr Psychiatry. 2006;47(1):35-41.

5. Cuijpers P, Smit F, Oostenbrink J, de Graaf R, Ten Have M, Beekman A. Economic costs of minor depression: a population-based study. Acta Psychiatr Scand. 2007;115(3):229-236.

6. Cuijpers P, Smit F. Subthreshold depression as a risk indicator for major depressive disorder: a systematic review of prospective studies. Acta Psychiatr Scand. 2004;109(5):325-331.

7. National Collaborating Centre for Mental Health. Depression: The Treatment and Management of Depression in Adults (Updated Edition). NICE Clinical Guidelines, No. 90. Leicester, United Kingdom: British Psychological Society; 2010.

8. Lewinsohn PM, Sullivan JM, Grosscup SJ. Changing reinforcing events: An approach to the treatment of depression. Psychother Theor Res Pract. 1980;17(3):322-334.

9. Cuijpers $P$, van Straten A, Warmerdam L. Behavioral activation treatments of depression: a meta-analysis. Clin Psychol Rev. 2007; 27(3):318-326.

10. Ekers D, Richards D, Gilbody S. A meta-analysis of randomized trials of behavioural treatment of depression. Psychol Med. 2008; 38(5):611-623.

11. Mazzucchelli T, Kane R, Rees C. Behavioral activation treatments for depression in adults: A meta-analysis and review. Clin Psychol Sci Pract. 2009;16(4):383-411.

12. Ekers D, Webster L, Van Straten A, Cuijpers P, Richards D, Gilbody S. Behavioural activation for depression; an update of meta-analysis of effectiveness and sub group analysis. PLoS One. 2014;9(6):e100100.

13. Ekers D, Richards D, McMillan D, Bland JM, Gilbody S. Behavioural activation delivered by the non-specialist: phase II randomised controlled trial. Br J Psychiatry. 2011;198(1):66-72.

14. Richards DA, Ekers D, McMillan D, et al. Cost and Outcome of Behavioural Activation versus Cognitive Behavioural Therapy for Depression (COBRA): a randomised, controlled, non-inferiority trial. Lancet. 2016;388(10047):871-880.

15. Martell CR, Addis ME, Jacobson NS. Depression in Context: Strategies for Guided Action. New York, NY: WW Norton \& Co; 2001.

16. Martell CR, Dimidjian S, Herman-Dunn R. Behavioral Activation for Depression: A Clinician's Guide. New York, NY: Guilford Press; 2010. 
17. Mazzucchelli TG. Behavioural Activation Interventions for Depression and Well-Being [thesis]. Perth, Western Australia: Curtin University of Technology; 2010.

18. Wong SY, Kung K, Griffiths SM, et al. Comparison of primary care experiences among adults in general outpatient clinics and private general practice clinics in Hong Kong. BMC Public Health. 2010; 10(1):397.

19. Hybels CF, Blazer DG, Pieper CF. Toward a threshold for subthreshold depression: an analysis of correlates of depression by severity of symptoms using data from an elderly community sample. Gerontologist. 2001;41(3):357-365.

20. Kroenke K, Spitzer RL. The PHQ-9: a new depression diagnostic and severity measure. Psychiatr Ann. 2002;32(9):509-515.

21. Zhang YL, Liang W, Chen ZM, et al. Validity and reliability of Patient Health Questionnaire-9 and Patient Health Questionnaire-2 to screen for depression among college students in China. Asia Pac Psychiatry. 2013;5(4):268-275.

22. Guest G, Bunce A, Johnson L. How many interviews are enough? An experiment with data saturation and variability. Field Methods. 2006;18(1):59-82

23. Hepner KA, Stern S, Paddock SM, Hunter SB, Osilla KC, Watkins KE. A fidelity coding guide for a group cognitive behavioral therapy for depression. TR- 980-NIDA/NIAAA. Santa Monica, CA: RAND Corporation; 2011.

24. Shek DT. Reliability and factorial structure of the Chinese version of the Beck Depression Inventory. J Clin Psychol. 1990;46(1):35-43.

25. Steer RA, Kumar G, Ranieri WF, Beck AT. Use of the Beck Depression Inventory-II with adolescent psychiatric outpatients. J Psychopathol Behav Assess. 1998;20(2):127-137.

26. Joiner TE Jr, Walker RL, Pettit JW, Perez M, Cukrowicz KC. Evidencebased assessment of depression in adults. Psychol Assess. 2005; 17(3):267-277

27. Kam I. Development of the Bilingual (Chinese/English) SCID-I (Struc tured Clinical Interview for DSM-IV Axis I Disorder): A Study of its Reliability and Validity in an In-Patient Population [dissertation]. Hong Kong, China: Hong Kong College of Psychiatrists; 2000.

28. Lam CL, Tse EY, Gandek B. Is the standard SF-12 health survey valid and equivalent for a Chinese population? Qual Life Res. 2005;14(2): 539-547.

29. Lee S, Tsang A, Huang Y-Q, et al. The epidemiology of depression in metropolitan China. Psychol Med. 2009;39(5):735-747.

30. Shek DT. The Chinese version of the State-Trait Anxiety Inventory: its relationship to different measures of psychological well-being. J Clin Psychol. 1993;49(3):349-358.

31. Cohen J. Statistical Power Analysis for the Behavioral Sciences. New York, NY: Routledge Academic; 1988.

32. Lakens D. Calculating and reporting effect sizes to facilitate cumulative science: a practical primer for t-tests and ANOVAs. Front Psychol. 2013;4:863.
33. Cohen J. A power primer. Psychol Bull. 1992;112(1):155-159.

34. Buuren S, Groothuis-Oudshoorn K. mice: Multivariate imputation by chained equations in R. J Stat Softw. 2011;45(3):1-67.

35. Rubin DB. Multiple Imputation for Nonresponse in Surveys. Hoboken, NJ: John Wiley \& Sons; 2004.

36. Sedgwick P. Per protocol analysis. BMJ. 2010;340:1825.

37. Button KS, Kounali D, Thomas L, et al. Minimal clinically important difference on the Beck Depression Inventory-II according to the patient's perspective. Psychol Med. 2015;45(15):3269-3279.

38. Linde K, Sigterman K, Kriston L, et al. Effectiveness of psychological treatments for depressive disorders in primary care: systematic review and meta-analysis. Ann Fam Med. 2015;13(1):56-68.

39. Cuijpers P, Smit F, Willemse G. Predicting the onset of major depression in subjects with subthreshold depression in primary care: a prospective study. Acta Psychiatr Scand. 2005;111(2):133-138.

40. Cuijpers P, Beekman A, Smit F, Deeg D. Predicting the onset of major depressive disorder and dysthymia in older adults with subthreshold depression: a community based study. Int J Geriatr Psychiatry. 2006;21(9):811-818.

41. Klein DN, Shankman SA, Lewinsohn PM, Seeley JR. Subthreshold depressive disorder in adolescents: predictors of escalation to fullsyndrome depressive disorders. J Am Acad Child Adolesc Psychiatry. 2009;48(7):703-710.

42. Bot M, Pouwer F, Ormel J, Slaets JP, de Jonge P. Predictors of incident major depression in diabetic outpatients with subthreshold depression. Diabet Med. 2010;27(11):1295-1301.

43. Hill RM, Pettit JW, Lewinsohn PM, Seeley JR, Klein DN. Escalation to major depressive disorder among adolescents with subthreshold depressive symptoms: evidence of distinct subgroups at risk. J Affect Disord. 2014;158:133-138

44. Garland EL, Fredrickson B, Kring AM, Johnson DP, Meyer PS, Penn DL. Upward spirals of positive emotions counter downward spirals of negativity: insights from the broaden-and-build theory and affective neuroscience on the treatment of emotion dysfunctions and deficits in psychopathology. Clin Psychol Rev. 2010;30(7): 849-864.

45. Nyklícek I, Kuijpers KF. Effects of mindfulness-based stress reduction intervention on psychological well-being and quality of life: is increased mindfulness indeed the mechanism? Ann Behav Med. 2008;35(3):331-340.

46. Grossman P, Niemann L, Schmidt S, Walach H. Mindfulness-based stress reduction and health benefits. A meta-analysis. J Psychosom Res. 2004:57(1):35-43.

47. Kanter JW, Santiago-Rivera AL, Santos MM, et al. A randomized hybrid efficacy and effectiveness trial of behavioral activation for Latinos with depression. Behav Ther. 2015;46(2):177-192.

48. Snarski M, Scogin F, DiNapoli E, Presnell A, McAlpine J, Marcinak J. The effects of behavioral activation therapy with inpatient geriatric psychiatry patients. Behav Ther. 2011;42(1):100-108. 